\title{
Enhancement of Maximum Time Delay in one Fiber Segment Slow Light Systems Based on Stimulated Brillouin Scattering
}

\author{
Ronny Henker \\ Deutsche Telekom Fachhochschule Leipzig \\ Thomas Schneider \\ Deutsche Telekom Fachhochschule Leipzig \\ Markus Junker \\ Deutsche Telekom Fachhochschule Leipzig
}

See next page for additional authors

Follow this and additional works at: https://arrow.tudublin.ie/engschececon

Part of the Electrical and Computer Engineering Commons

\section{Recommended Citation}

Henker, R. et al. (2007) Enhancement of maximum time delay in one fiber segment slow light systems based on stimulated Brillouin scattering. CLEO 2007. Conference on Lasers and Electro-Optics, pp.1-2. Baltimore, 6-11 May, 2007, doi:10.1109/CLEO.2007.4452358

This Conference Paper is brought to you for free and open access by the School of Electrical and Electronic Engineering at ARROW@TU Dublin. It has been accepted for inclusion in Conference papers by an authorized administrator of ARROW@TU Dublin. For more information, please contact arrow.admin@tudublin.ie, aisling.coyne@tudublin.ie,gerard.connolly@tudublin.ie.

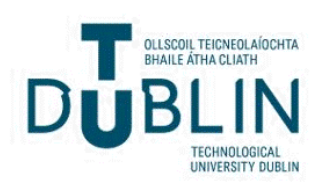




\section{Authors}

Ronny Henker, Thomas Schneider, Markus Junker, Kai-Uwe Lauterbach, Max Ammann, and Andreas Schwarzbacher 


\title{
Enhancement of maximum time delay in one fiber segment slow light systems based on stimulated Brillouin scattering
}

\author{
Ronny Henker, Thomas Schneider, Markus Junker, Kai-Uwe Lauterbach \\ Deutsche Telekom AG, Fachhochschule Leipzig, Gustav-Freytag Str. 43-45, D-04277 Leipzig, Germany \\ ronny.henker@fh-telekom-leipzig.de \\ Max James Ammann, Andreas Thomas Schwarzbacher \\ School of Electronic \& Communications Engineering, Dublin Institute of Technology, Kevin Street Dublin 8, Ireland
}

\begin{abstract}
An effective method to enhance the time delay in SBS-based slow-light systems by decoupling the delay from the Brillouin gain is shown. A drastic improvement of the time delay in one fiber segment was achieved.

(C)2007 Optical Society of America

OCIS codes: (999.9999) Slow light; (290.5900) Scattering, stimulated Brillouin
\end{abstract}

Slow and fast light has attracted an increasing interest during the last years because it offers the potential for an all optical control of the velocity of light pulses. Especially the nonlinear effect of stimulated Brillouin scattering (SBS) has become an effective and flexible tool for the development of a number of very interesting applications in optical telecommunications and optical signal processing [1]. In [2] an extremely wide group velocity control as small as $71000 \mathrm{~km} / \mathrm{s}$ and even negative group velocities were observed in a short optical fiber.

But one problem of the SBS is that in most experiments the maximum pulse delay is limited to around $30 \mathrm{~ns}$ $[1,3]$. Most SBS-based slow light systems presented so far can be seen as a Brillouin amplifier for which the amplifier gain is the logarithmical relation between the optical power of the delayed pulse at the output $P_{D}(L)$ and the input $P_{D}(0)$ of the amplifier $[4,5]$ :

$$
G_{d B}=10 \log \left(\frac{P_{D}(L)}{P_{D}(0)}\right) \approx 4.343 g_{0} \frac{P_{P} L_{e f f}}{A_{\text {eff }}} \approx 4.343 \Delta f_{B} 2 \pi \Delta T .
$$

where $g_{0}$ is the line center SBS gain coefficient, $P_{P}$ the pump power, $\Delta f_{B}$ the Brillouin line width, $A_{\text {eff }}$ and $L_{\text {eff }}$ the effective area and length of the fiber. As can be seen from Eq. (1) the time delay $\Delta T$ increases with higher optical powers of the pump source. This is always accompanied by a higher amplifier gain and an unavoidable amplification of the delayed pulses. But if the amplification becomes too high the delay goes over into a saturation regime because the pump depletion and the amplified spontaneous Brillouin scattering limits further delay times.

One opportunity to enhance the maximum time delay is to cascade several delay lines [6] but this method is very complicated because additional equipment is required and the properties of all fiber segments have to be identical referring to the SBS. Another method which is much easier and more effective is to decouple the amplifier gain and the time delay [5, 7]. This is possible if the base line of the SBS gain is shifted into the loss region. That causes a reduction of $g_{0}$ whereas the shape of the Brillouin gain will remain the same in the frequency domain. Since the group velocity and hence the time delay is a function of the group index in the fiber - which in turn depends on the shape of the Brillouin gain - the time delay will also be unchanged. However, the amplifier gain is a function of the absolute height of the Brillouin gain and so the pump depletion is reduced. This results in higher usable pump powers and hence in higher time delays. The method can be achieved by superimposing a SBS gain $+G_{l}$ with the bandwidth $\triangle f_{B I}$ and a SBS loss $-G_{2}$ with the bandwidth $\triangle f_{B 2}$. The resulting delay is therefore [5]:

$$
\Delta T_{\text {res }}=\frac{G_{1}}{2 \pi \Delta f_{B 1}}-\frac{G_{2}}{2 \pi \Delta f_{B 2}}
$$

where $G_{l, 2}=g_{0} P_{P l, 2} L_{e f f} / A_{\text {eff. }}$. In contrast to [5] with our method we achieved a maximum time delay which is around six times higher.

Our principle experimental setup for this method is shown in Fig. 1. We used two distributed feedback (DFB) pump lasers at a wavelength of $1550 \mathrm{~nm}$ to generate a Brillouin spectrum in a $50 \mathrm{~km}$ SSMF. Due to such a very long fiber the required pump powers can be minimized. Each pump source creates a gain at $f_{P}-f_{B}$ and a loss at $f_{P}+f_{B}$ via Brillouin scattering, with $f_{P}$ as the optical frequency of the lasers and $f_{B}$ as the natural Brillouin shift in the fiber, which is typically at around $11 \mathrm{GHz}$ in a SSMF. By adjusting $f_{P I}=f_{P 2}+2 f_{B}$ the gain spectrum of the first pump laser (Pump1) superimposes with the loss of the second (Pump2). Thereby, the loss spectrum is broadened by a direct modulation of the laser with a noise signal and the power of the loss and gain spectrum is additionally controlled by an erbium-doped fiber amplifier (EDFA) and a tunable optical attenuator (TOA). The two pump waves are coupled into the SSMF via a circulator (C). From the other side a pulse signal, which is produced by a narrow bandwidth 


\section{CFD4.pdf}

$(<1 \mathrm{kHz})$ fiber laser and a Mach-Zehnder modulator $(\mathrm{MZM})$ driven by a waveform generator, is coupled into the same fiber. The pulses had a wavelength of $1550 \mathrm{~nm}$, a repetition rate of $1 \mathrm{MHz}$ and a temporal duration of around $35 \mathrm{~ns}$. In the fiber the $25 \mathrm{~dB}$ attenuated (Att) pulses will be amplified and delayed by the coincided loss and gain spectrum; finally detected by a photodiode (PD) and interpreted by an oscilloscope (Osci) at port 3 of the circulator.

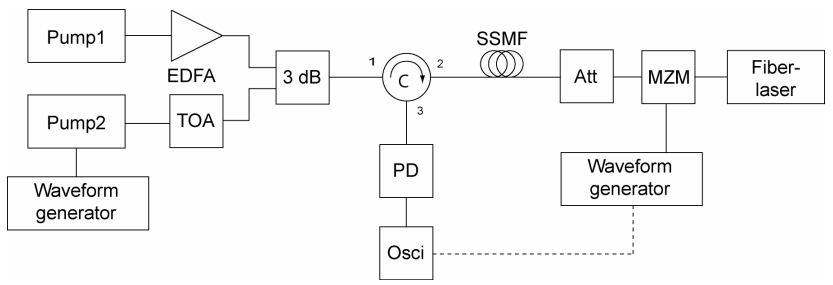

Fig. 1. Principle experimental setup.

The results for a pulse delay without and with additional loss spectrums are shown in Fig. 2. For small rising pump powers of Pump1 the delay increases linearly for all conditions. Thus, the time delay does not change by adding the loss spectrum at first. Then, the time delay reaches the saturation regime caused by the pump depletion; without a loss spectrum at around $35 \mathrm{~ns}$. But, by adding the loss spectrum with a 3-dB bandwidth of around $180 \mathrm{MHz}$ the pump depletion is reduced. It can be seen that with higher pump powers of the gain laser the saturation regime is reached later; with a loss spectrum of $10.5 \mathrm{~mW}$ at around $55 \mathrm{~ns}$. Thus, increasing pump powers of the loss laser results in higher maximum time delays. Without the loss spectrum we achieved a maximum time delay of only approximately $55 \mathrm{~ns}$ for a gain pump power of $20 \mathrm{~mW}$ whereas for the loss spectrums of $4 \mathrm{~mW}, 9 \mathrm{~mW}$ and $10.5 \mathrm{~mW}$ delays of $60 \mathrm{~ns}, 67 \mathrm{~ns}$ and $70 \mathrm{~ns}$ occurred which is an unprecedented enhancement of $27 \%$. Nevertheless, the time delay can be further increased up to around $100 \mathrm{~ns}$ with a higher loss in our method [7].

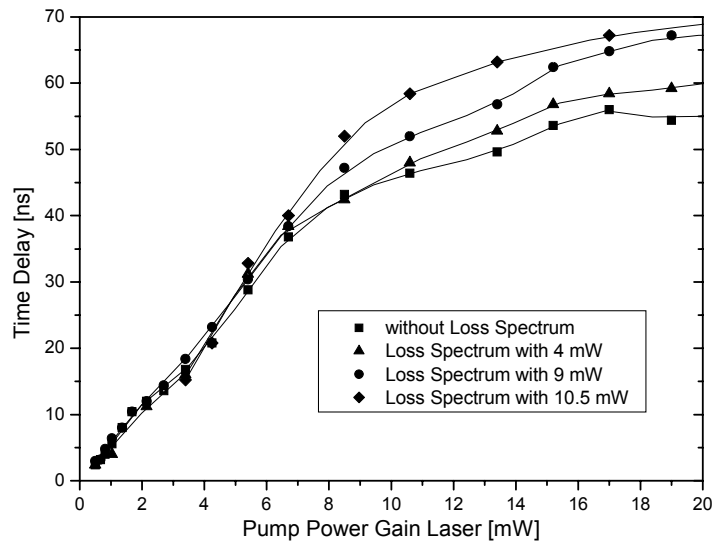

Fig. 2. Time delay against the gain laser pump power with and without an additional loss spectrum.

In conclusion we have shown how the maximum time delay can be enhanced by decoupling the delay from the Brillouin gain in a one fiber segment SBS-based slow light system. By variably reducing the line center SBS gain coefficient with an additional loss spectrum a continued adjustment above the common time delay of $30 \mathrm{~ns}$ is possible. With this simple and flexible method we achieved maximum pulse delays of $70 \mathrm{~ns}$ in a $50 \mathrm{~km} \mathrm{SSMF}$.

We gratefully acknowledge the help of J. Klinger of Fachhochschule Leipzig.

\section{References}

[1] Y. Okawachi, M. S. Bigelow, J. E. Sharping, Z. Zhu, A. Schweinsberg, D. J. Gauthier, R. W. Boyd, and A. L. Gaeta, "Tunable alloptical delays via Brillouin slow light in an optical fiber,” Phys. Rev. Lett. 94, 153902 (2005).

[2] M. G. Herráez, K. Y. Song, and L. Thévenaz, "Optically controlled slow and fast light in optical fibers using stimulated Brillouin scattering," Appl. Phys. Lett. 87, 081113 (2005).

[3] K. Y. Song, M. G. Herráez, and L. Thévenaz, "Observation of pulse delaying and advancement in optical fibers using stimulated Brillouin scattering," Opt. Express 13, 82-88 (2005).

[4] Th. Schneider, Nonlinear Optics in Telecommunications (Springer-Verlag, 2004)

[5] S. Chin, M. G. Herráez, and L. Thévenaz, "Zero-gain slow \& fast light propagation in an optical fiber," Opt. Expres 14, 10684 (2006).

[6] K. Y. Song, M. G. Herráez, and L. Thévenaz, "Long optically controlled delays in optical fibers," Opt. Lett. 30, 1782-1784 (2005).

[7] Th. Schneider, M. Junker, and K.-U. Lauterbach, "Time delay enhancement in stimulated Brillouin scattering based slow light systems," Opt. Lett. 32, (2007) 